\title{
Serum lipids and glucose concentrations in subjects using antihypertensive drugs: Finnmark 1977
}

\author{
EGIL ARNESEN, DAG S THELLE, OLAV H FøRDE, AND OLE D MJøS \\ From the Institute of Community Medicine and the Institute of Medical Biology, University of Troms $\phi$, \\ Norway
}

SUMMARY As'a side project to a study of coronary risk factors 4878 men and women aged 20-53 were interviewed about present and previous use of antihypertensive drugs. Serum lipid and glucose concentrations were compared in 124 present users, 73 previous users, and 124 controls matched for age, sex, and systolic and diastolic blood pressure. Users of betablockers, thiazides, and other antihypertensive drugs had higher total cholesterol, triglycerides, and glucose and lower HDL-cholesterol than the other groups, but only the difference in HDL-cholesterol was statistically significant. Smokers had statistically significant lower HDL-cholesterol than non-smokers in drug users, whereas there were only minor differences between them in previous and never users. This indicates an interaction between smoking and current antihypertensive medication. The unfavourable serum lipid pattern may, if caused by drug use, explain the lack of influence that antihypertensive treatment has had on the incidence of coronary heart disease in intervention studies.

Drug treatment of hypertension has often failed to decrease the incidence of coronary heart disease and total mortality. ${ }^{12} \mathrm{~A}$ possible explanation for the lack of effect on the incidence of coronary heart disease could be unfavourable metabolic effects of the medication. Such unfavourable changes in serum lipids have been reported both from clinical trials ${ }^{3-8}$ and epidemiological studies. ${ }^{9-11}$ In the cardiovascular survey in Finnmark carried out in $1974-5^{\circ}$ subjects who reported that they were having treatment for hypertension had higher cholesterol, triglycerides, and glucose than "healthy" subjects. These findings initiated a side project to the cardiovascular survey in Finnmark 1977 where information was obtained on present and previous use of antihypertensive drugs. In this paper we compare serum lipid concentrations in current users, previous users, and never users of such medication.

\section{Methods and material}

All subjects aged $20-53$ in four municipalities in Finnmark were invited to the screening. Of 6088 invited, $4878(80 \cdot 1 \%)$ attended. The procedure and methods, except for high-density lipoprotein cholesterol (HDL-C) determination, were identical to those used in the former cardiovascular study in Finnmark and have been described elsewhere. ${ }^{12}$ At the screening specially trained interviewers asked each subject the following two questions: "Do you use antihypertensive drugs?" and "Have you previously used antihypertensive drugs?" If the answer to any of these questions was "yes" the participant was asked which brand(s) was used. If the participant was a former user the year of stopping was recorded. All subjects currently using nitroglycerine, quinidine, digitalis, and drugs of unknown brands were excluded. The subjects were divided into those who used alprenolol, propranolol, and pindolol (betablockers) and those who used hydrochlorthiazide, trichlormethiazide, and polythiazide (thiazides) and clonidine, chlorthalidone, and frusemide (called other).

\section{HDL - C DETERMINATION}

The serum samples, drawn in a non-fasting state, were stored at $+4^{\circ} \mathrm{C}$ and analysed at the Institute of Medical Biology in Troms $\varnothing$ within two weeks. HDL-C was assayed enzymatically after precipitation of lipoprotein with density $<1.063$ with heparin and $\mathrm{MnCL}_{2}$ by the method of Burstein et al. ${ }^{13}$ The modifications are described elsewhere. ${ }^{14}$ The coefficient of variation for replicated measurements of HDL-C was $2 \cdot 7 \%$.

\section{STATISTICAL METHODS}

Age and sex adjustments were made according to the formula $\mathrm{z}=(\mathrm{Xi}-\overline{\mathrm{X}}) / \mathrm{SD}$, where $\mathrm{Xi}$ is the subject's 
observed value, $\overline{\mathrm{X}}$ the mean value for the subject's sex and five-year group in the total population who attended and were examined, and SD the standard deviation of this group. $\mathrm{Z}$ denotes the subject's relative position within his own sex and age group measured in standard deviation units. Each current user of an antihypertensive drug was matched on sex and age (10-year groups) with a single control who had never used these drugs and who had systolic and diastolic blood pressure within one SD of the adjusted mean of user group.

Adjustment for differences in relative weight $\left(\mathrm{g} / \mathrm{cm}^{2}\right)$ between groups was done according to the direct method $^{15}$ with matched never users as the reference population.

Statistical significance of differences between adjusted means were estimated by using a two-tailed $t$ test.

\section{MATERIAL}

Altogether 124 subjects (51 men and 73 women) were current users and 73 subjects ( 25 men and 48 women) were previous users. Mean systolic and diastolic blood pressure of current users were 152.7 and $97.4 \mathrm{~mm} \mathrm{Hg}$, of previous users 146.0 and $93.4 \mathrm{~mm} \mathrm{Hg}$, and of never users 151.2 and $96 \cdot 1 \mathrm{~mm} \mathrm{Hg}$.

\section{Results}

Table 1 shows the unadjusted means and standard deviations and table 2 the mean $z$-values of serum lipids and glucose in the user categories. A z-value of 0.22 for total cholesterol in matched never users denotes that this group of 124 has a cholesterol level of 0.22 standard deviations or approximately $0.31 \mathrm{mmol} / 1(1.40 \times 0.22)$ above the mean for the total population adjusted for age and sex. This differs significantly from $z=0(p<0 \cdot 05)$.
Subjects using antihypertensive drugs had significantly lower HDL-C and relative HDL-C compared both with previous and never users $(p<0 \cdot 01)$. These differences remained significant after adjustment for relative weight. Between users of different drug categories and between previous users and never users no such differences were observed. There was a trend towards higher cholesterol, triglyceride, and glucose levels in users, but those differences were not statistically significant. The material was also analysed for cigarette smokers and non-smokers separately. This analysis showed larger differences as the $\mathrm{z}$-values for HDL-C in smokers were $-0.73,-0.01$, and -0.01 for the users, previous users, and never users respectively $(p<0 \cdot 005)$. Among the non-smokers the HDL-C differences were somewhat reduced $(-0.36,-0.08$, and 0.07 , respectively) but remained significant between the users and never users $(p<0 \cdot 01)$. There was a statistically significant difference between smoking and non-smoking drug users $(\mathrm{p}<0.02)$. For the other variables, except for relative HDL-C which followed the HDL-C, the analysis within smoking categories showed the same pattern as for the total group.

\section{Discussion}

In cross-sectional studies one can never completely rule out the possibility that the observed differences are caused by selection bias and confounding variables rather than particular treatment. In the present study, however, a considerable part of the bias appears to have been removed by the use of a blood pressure matched control group. All never users of antihypertensive drugs, adjusted for age and $\operatorname{sex}(z=0, S D=1)$, differ clearly in total cholesterol from the drug users $(z=0.27, p<0 \cdot 01)$. On the other hand, the control group had a small $(z=0 \cdot 22)$

Table 1 Mean and standard deviation for total cholesterol, high-density lipoprotein cholesterol, relative HDL-C,* triglyceride, and blood glucose according to drug use, Finnmark 1977

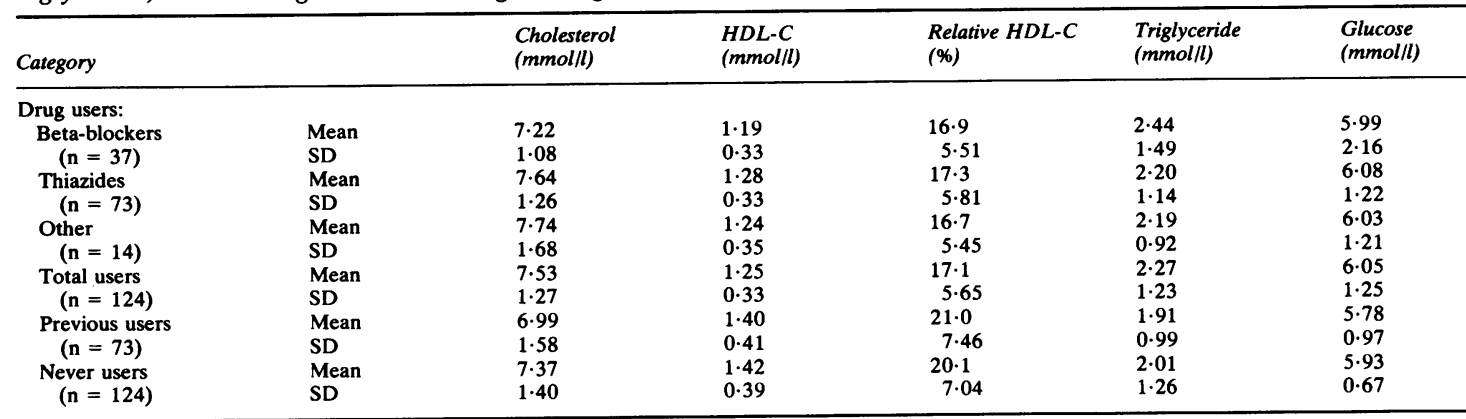

*Relative HDL-C $=$ HDL-C as a percentage of total cholesterol. 
Table 2 Z-value (mean and standard deviation) for total cholesterol, high-density lipoprotein cholesterol, relative HDL-C,* triglycerides and blood-glucose according to drug use, Finnmark 1977

\begin{tabular}{|c|c|c|c|c|c|c|}
\hline Category & & Cholesterol & $H D L-C$ & Relative $H D L-C$ & Triglycerides & Glucose \\
\hline \multicolumn{7}{|l|}{ Drug users: } \\
\hline \multirow[t]{2}{*}{ Beta-blockers } & Mean & $0 \cdot 12$ & -0.51 & -0.51 & 0.47 & 0.07 \\
\hline & SD & 0.75 & 0.75 & 0.67 & 1.27 & 1.88 \\
\hline \multirow[t]{2}{*}{ Thiazides } & Mean & 0.33 & -0.45 & -0.52 & 0.36 & 0.33 \\
\hline & SD & 0.97 & 0.87 & 0.85 & $1 \cdot 25$ & $1 \cdot 39$ \\
\hline \multirow[t]{2}{*}{ Other } & Mean & 0.38 & -0.56 & -0.62 & 0.30 & 0.34 \\
\hline & SD & $1 \cdot 33$ & 0.90 & 0.73 & 0.98 & 1.46 \\
\hline \multirow[t]{2}{*}{ Total users } & Mean & 0.27 & -0.48 & -0.52 & 0.38 & 0.25 \\
\hline & SD & 0.96 & 0.83 & 0.79 & $1 \cdot 22$ & $1 \cdot 55$ \\
\hline \multirow[t]{2}{*}{ Previous users } & Mean & $0 \cdot 18$ & -0.05 & $-0 \cdot 11$ & $0 \cdot 16$ & $0 \cdot 10$ \\
\hline & SD & $1 \cdot 17$ & $1 \cdot 10$ & 1.01 & 0.97 & $1 \cdot 19$ \\
\hline \multirow[t]{2}{*}{ Never users } & Mean & 0.22 & 0.03 & -0.11 & 0.14 & 0.16 \\
\hline & SD & 1.02 & 1.03 & 1.01 & $1 \cdot 20$ & 0.74 \\
\hline
\end{tabular}

Relative HDL-C $=$ HDL-C as a percentage of total cholesterol.

non-significant difference from drug users $(z=0 \cdot 27)$. This pronounced effect of the matching for blood pressure indicates that the total cholesterol difference observed in the first Finnmark survey ${ }^{9}$ may well have been caused by confounding factors.

Confounding factors may remain in this study even after the blood pressure matching. A few variables have been explicitly examined from this point of view. For instance, the analysis within smoking categories gave larger differences, indicating an interaction between antihypertensive medication and cigarette smoking. Adjustment for relative weight reduced the differences somewhat but not enough to alter the significance of the findings. One must, however, keep in mind the potential effect of alcohol consumption and physical activity has not been controlled for in the present analysis. An indication of remaining confounding factors may be the similarity between users of different drug categories, which was surprising on the background of the diversity in chemical composition and mode of action of those drugs. In variables other than cholesterol the blood pressure matching had a much smaller effect, especially in HDL-C where the matched never users had a $\mathrm{z}$-value close to the total group of never users. In addition HDL-C was the only variable where drug users differ most from never users. A reduced HDL-C in users of beta-blockers has been seen in other studies, ${ }^{4511}$ whereas a similar effect of thiazide has not been reported. Helgeland $e t$ $a l^{4}$ reported no significant difference in HDL-C between thiazide users and controls and neither did Grimm $e t \mathrm{al}^{7}$ in a double-blind crossover study. In the LRC study ${ }^{11}$ a trend in the opposite direction toward higher HDL-C in thiazide users was observed, especially for women. For total cholesterol no statistically significant differences were observed in users, previous users, and matched never users. These results are in accordance with other reports that show none or minor changes in total cholesterol $^{3-5,7-8}$ for most antihypertensive drugs.

The difference in HDL-C between smokers and non-smokers was much larger in drug users than among never users. This interaction may indicate use of stronger indications for antihypertensive medication among smokers. Drug users had altogether the most unfavourable level for all the compared variables in the present study. If the observed differences in serum lipids are caused by the drug use the unfavourable effect may be large enough to outweigh a beneficial effect of blood pressure reduction on the incidence of coronary heart disease.

Requests for reprints to: Egil Arnesen, Institute of Community Medicine, N-9010 Åsgård sykehus, Norway.

\section{References}

${ }^{1}$ Veterans administration cooperative study group on antihypertensive agents. Effect of treatment on morbidity in hypertension. II. Results in patients with diastolic blood pressure averaging 90 through $114 \mathrm{~mm} \mathrm{Hg}$. JAMA 1970; 213: 1143-52.

${ }^{2}$ US public health service hospitals cooperative study group-McFate Smith, W. Treatment of mild hypertension. Results of a 10-year intervention trial. Circ Res 1977; 40, suppl 1: 98-105.

${ }^{3}$ Berglund G, Andersson O. Beta-blockers or diuretics in hypertension? A six-years follow-up of blood pressure and metabolic side effects. Lancet 1981; i: 744-7.

'Helgeland A, Hjermann I, Leren P, Enger S, Holme I. High-density lipoprotein cholesterol and antihypertensive drugs: the Oslo study. $\mathrm{Br}$ Med J 1978; ii: 403.

${ }^{5}$ Leren P, Foss OP, Helgeland A, Hjermann I, Holme I, Lund-Larsen PG. Effect of propranolol and prazosin on blood lipids. The Oslo study. Lancet 1980; ii: 4-6.

- Lewis PJ, Kohner EM, Petrie A, Dollery CT. Deterioration of glucose tolerance in hypertensive patients on prolonged diuretic treatment. Lancet 1976; i: $564-6$. 
${ }^{7}$ Grimm RH, Leon AS, Hunninghake D, Lenz K, Hannan $P$, Blackburn $H$. Effects of thiazide diuretics on plasma lipids and lipoproteins in mildly hypertensive patients. Ann Intern Med 1981; 94: 7-11.

${ }^{8}$ Shaw J, England JDF, Hua ASP. Beta-blockers and plasma triglyceride. $\mathrm{Br}$ Med J 1978; i: 986.

${ }^{9}$ National Mass Radiography Service, Health Services of Finnmark County, Central Laboratory, Ullevål Hospital and Faculty of Medicine, University of Troms $\emptyset$. The cardiovascular study in Finnmark 1974-75. Nordic Council Arctic Medical Research Report 1979; 25: 1-191.

${ }^{10}$ Leren P, Askevold E-M, Foss OP, et al. The Oslo study. Cardiovascular disease in middle-aged and young Oslo men. Acta Med Scand 1975; 588, suppl: 1-38.

11 Wallace RB, Hunninghake DB, Reiland S, et al. Alterations of plasma high-density lipoprotein cholesterol levels associated with consumption of selected medications. Circulation 1980; 62, suppl IV: 77-82.
${ }^{12}$ Bjartveit K, Foss OP, Gjervig T, Lund-Larsen PG. The cardiovascular disease study in Norwegian counties. Acta Med Scand suppl 1979; 634: 1-70.

${ }^{13}$ Burstein M, Scholnich HR, Morfin R. Rapid method for the isolation of lipoproteins from human serum by precipitation with polyanions. J Lipid Res 1970; 11: 583-5.

${ }^{14}$ Førde OH, Thelle DS, Miller NE, Mjøs OD. The Troms $\varnothing$ heart study. Distribution of serum cholesterol between high density and lower density lipoproteins in subjects of Norse, Finnish and Lappish ethnic origin. Acta Med Scand 1978; 203: 21-6.

${ }^{15}$ Remington RD, Schork MA. Statistics with applications to the biological and health sciences. New Jersey: Prentice-Hall Inc, 1970. 